\section{THU0400 INCIDENCE AND RISK FACTORS FOR GANGRENE IN PATIENTS WITH SYSTEMIC SCLEROSIS FROM THE EUSTAR COHORT}

${ }^{1,2}$ C. Mihai, O. Distler ${ }^{1}$, A.M. Gheorghiu' ${ }^{2}$, P.I. Constantin2, R. Dobrota ${ }^{1,2}$, S. Jordan ${ }^{1}$, V. Smith ${ }^{3,4}$, E. Hachulla ${ }^{5}$, J. Henes ${ }^{6}$, E. Siegert ${ }^{7}$, S. Vettori ${ }^{8}$, U. MüllerLadner $^{9}$, M. Matucci-Cerinic ${ }^{10}$, Y. Allanore ${ }^{11}$, on behalf of EUSTAR. ${ }^{1}$ Department of Rheumatology, Zurich University Hospital, Zurich, Switzerland; ${ }^{2}$ Internal Medicine and Rheumatology, Cantacuzino Hospital, Carol Davila University of Medicine and Pharmacy, Bucharest, Romania; ${ }^{3}$ Department of Rheumatology, Ghent University Hospital; ${ }^{4}$ Department of Internal Medicine, Ghent University, Ghent, Belgium; ${ }^{5}$ Department of Internal Medicine and Clinical immunology, Referral Centre for Rare Systemic Auto-immune Diseases North and North-West of France, University of Lille, Huriez' Hospital, Lille, France; ${ }^{6}$ Department of Rheumatology, Tübingen University Hospital, Tübingen; ${ }^{7}$ Department of Rheumatology, Charité University Hospital, Berlin, Germany; ${ }^{8}$ Rheumatology Unit, Internal and Experimental Medicine, Second University of Naples, II Policlinico, Naples, Italy; ${ }^{9}$ Department of Rheumatology and Clinical Immunology, JustusLiebig University Giessen, Campus Kerckhoff, Bad Nauheim, Germany;

${ }^{10}$ Department of Experimental and Clinical Medicine, University of Florence, Florence, Italy, ${ }^{11}$ Department of Rheumatology, Cochin Hospital, Paris Descartes University, Paris, France

Background: Digital ulcers (DUs) affect about half of systemic sclerosis (SSc) patients during disease course. In some patients, peripheral vasculopathy can promote critical ischemia and gangrene, severe complications with potential life threatening consequences. Recently the DUO registry suggested a $18 \%$ prevalence of gangrene in DU-SSc patients, with smoking and a high number of DUs being predictive factors. However, little is known about gangrene in unselected SSc patients.

Objectives: To investigate the prevalence, incidence and risk factors for gangrene in the EUSTAR cohort.

Methods: We included patients from the EUSTAR database satisfying the ${ }^{\text {ACR } 1980}$ or the ACR/EULAR 2013 classification criteria for SSc, with at least one visit recording data on gangrene. We extracted from this database data regarding the reporting of DUs, DUs history and digital gangrene. Centres were asked for supplementary data on traditional cardiovascular (CV) risk factors. We analysed by uni- and multivariable logistic regression the cross-sectional relationship between gangrene and its potential risk factors such as history of DUs, cutaneous subset, disease duration, autoantibodies, traditional CV risk factors. Furthermore, longitudinal data were analysed by Cox proportional hazards regression.

Results: 1757 patients matched the inclusion criteria (age at inclusion $55.9 \pm 14.5$ years, disease duration since first non-Raynaud's symptom $7.9 \pm 10.3$ years and from onset of Raynaud's phenomenon (RP) $11.1 \pm 11.0$ years, male sex $16.7 \%$, $24.6 \%$ diffuse cutaneous subset (DcSSc)). At inclusion, $8.9 \%$ of patients had either current or previous digital gangrene, $15.7 \%$ had current DUs and a further $25.8 \%$ had previously had DUs. Among the potential risk factors, older age, a history of DUs and the DcSSc subset were statistically significant risk factors in the cross-sectional multivariable model.

For the longitudinal part, during the entire follow-up (median [Q1,Q3] 13.1 [9.6, 19.3] months), 16/771 patients had incident gangrene (2.1\%), accounting for an incidence of $1.73 / 100$ patient-years. All 16 patients who developed incident gangrene had previously had DUs and gangrene. Further risk factors for incident gangrene were the DcSSc subset and longer disease duration: hazard ratio [95\% confidence interval]: 8.97 [2.90-27.71] and 1.08 [1.04-1.13] respectively, both $\mathrm{p}<0.001$

Conclusions: In unselected SSc patients, gangrene still occurs in about $9 \%$ of SSc patients. Of the most importance, a history of DUs and, to a lesser extent, the DcSSc subset are strongly and independently associated with gangrene, while traditional CV risk factors were not identified as risk factors. Our results confirm that gangrene is still a concern in SSc. They emphasise the importance of microvascular SSc-associated disease in the pathogenesis of gangrene and suggest that the DcSSc subset should be prioritised for risk-stratification of the patients. Acknowledgements: The authors thank all the contributing EUSTAR investigators and patients.

Disclosure of Interest: C. Mihai Grant/research support from: Actelion Pharmaceuticals Ltd, Abbvie, Speakers bureau: Roche, Geneva Romfarm, O. Distler Grant/research support from: Actelion, Bayer, Boehringer Ingelheim, Mitsubishi Tanabe Pharma, Roche, Consultant for: Actelion, Bayer, Biogenldec, Boehringer Ingelheim, ChemomAb, espeRare foundation, Genentech/Roche, GSK, Inventiva, Italfarmaco, Lilly, medac, Medlmmune, Mitsubishi Tanabe Pharma, Pharmacyclics, Novartis, Pfizer, Sanofi, Sinoxa, A. M. Gheorghiu Grant/research support from: Geneva Romfarm, Abbvie, P. Constantin: None declared, R. Dobrota Grant/ research support from: Actelion Pharmaceuticals Ltd, Pfizer, S. Jordan: None declared, V. Smith Grant/research support from: Actelion Pharmaceuticals Ltd, Boehringer-Ingelheim Pharma GmbH, Bayer AG, Roche NV/SA, Fund for Scientific Research Flanders, E. Hachulla: None declared, J. Henes: None declared, E.
Siegert: None declared, S. Vettori: None declared, U. Müller-Ladner: None declared, M. Matucci-Cerinic: None declared, Y. Allanore Grant/research support from: BMS, Genentech-Roche, Inventiva, Pfizer, Sanofi, Consultant for: Actelion, Bayer, Biogen, Genentech-Roche, Galapagos, Medac, Pfizer, Sanofi, Servier, UCB

DOI: 10.1136/annrheumdis-2018-eular.4621

\section{THU0401 GLOBAL LONGITUDINAL STRAIN AS EARLY PREDICTOR OF SYSTOLIC DYSFUNCTION IN SYSTEMIC SCLEROSIS}

${ }^{1}$ C. Fischetti, F. Guerra ${ }^{2}$, A. Ferrarini ${ }^{3}$, L. Zuliani ${ }^{3}$, G. Stronati ${ }^{2}$, P. Fraticelli ${ }^{3}$, M. G. Danieli ${ }^{3}$, A. Capucci ${ }^{2}$, A. Gabrielli'. ${ }^{1}$ Dipartimento di Scienze Cliniche e Molecolari, Università Politecnica delle Marche; ${ }^{2}$ Department of Cardiology; ${ }^{3}$ Department of Internal Medicine, Azienda Ospedaliero Universitaria Ospedali Riuniti "Umberto I- Lancisi- Salesi" Ancona, Ancona, Italy

Background: Systemic sclerosis (SSc) is a chronic autoimmune disease of unknown etiology, characterised by microvascular abnormalities, immune abnormalities and progressive cutaneous and internal organs fibrosis. Subclinical heart disease in SSc patients is common but difficult to detect through conventional imaging.

Objectives: We sought to evaluate speckle-tracking derived global longitudina strain (GLS) as an early marker of subclinical systolic dysfunction in patients with SSc.

Methods: We enrolled 52 patients with SSc and 52 age and gender matched con trols. Patients with structural heart disease, heart failure, atrial fibrillation or pulmonary hypertension were excluded. An echocardiographic exam was performed for all patients, and standard and specke-tracking derived variables for the systolic and diastolic function of the left ventricle (LV) and right ventricle (RV) were acquired. SSc variant, antibodies pattern, cardiovascular risk factors and involvement of other organ systems were recorded.

Results: Common parameters of left and right systolic function did not differ between SSc patients and controls and were on average well above the cut-off for normality (all $p=N S$ ). LV and RV GLS were significantly impaired in patients with SSc when compared to healthy controls $(-19.2 \%$ vs. $-21.1 \% ; p=0.009$ and $-18.2 \%$ vs. $-22.3 \%$; $p=0.012$ respectively). In patients with SSc, GLS impairment was greater in basal segments when compared to midventricular and apical ones and homogeneous between the endo-, meso-, or epicardial layers of the RV, while LV showed an eccentric pattern with the epicardial layers mostly impaired Using $-20 \%$ as a cut-off for GLS, SSc patients had a 2.5 -fold increased risk of subclinical LV systolic impairment (OR 2.5; 95\% Cl 1.1-5.5; $p=0.027$ ) and a 3.3fold increased risk of subclinical RV systolic impairment when compared to age and gender matched controls (OR 3.3; 95\% Cl 1.4-7.7; $p=0.004$ ).

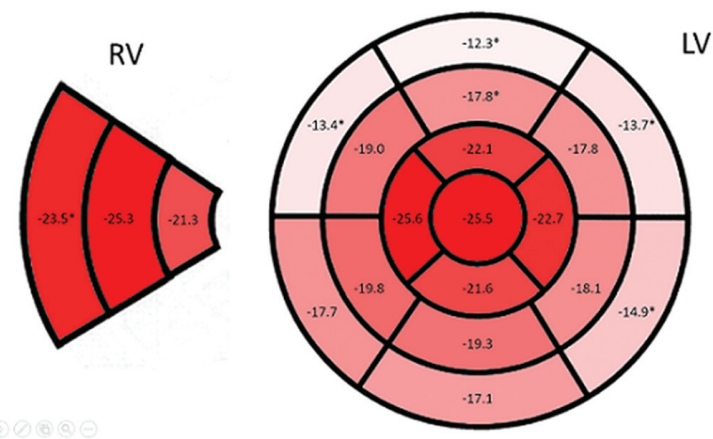

Conclusions: While traditional parameters are ineffective in detecting subclinical systolic impairment, a reduced GLS is common in patients with SSc and is significant for both LV and RV. While GLS impairment recognises a basal-apical gradient, transmural heart involvement seems different between RV and LV, suggesting a different mechanism of disease between the two ventricles.

Disclosure of Interest: None declared

DOI: 10.1136/annrheumdis-2018-eular.6423 